\title{
CIZ1 regulates the proliferation, cycle distribution and colony formation of RKO human colorectal cancer cells
}

\author{
JING YIN $^{1,2}$, CHANGLIANG WANG $^{3}$, XIAOYONG TANG ${ }^{4}$, HUAIBIN SUN $^{1}$, \\ QIANQIAN SHAO ${ }^{1}, \mathrm{XIGUI} \mathrm{YANG}^{4}$ and $\mathrm{XUN} \mathrm{QU}^{1}$ \\ ${ }^{1}$ Institute of Basic Medical Sciences, Qilu Hospital, Shandong University, Jinan, Shandong 250012; \\ ${ }^{2}$ Department of Internal Medicine Oncology, Affiliated Hospital of Shandong Academy of Medical Science, \\ Shandong Academy of Medical Sciences, Jinan, Shandong 250031; ${ }^{3}$ Department of Dermatology, \\ Shandong Provincial Institute of Dermatology and Venereology, Shandong Academy of Medical Sciences, \\ Jinan, Shandong 250022; ${ }^{4}$ Department of Internal Medicine Oncology, Shandong Cancer Hospital, \\ Shandong Academy of Medical Sciences, Jinan, Shandong 250117, P.R. China
}

Received April 24, 2013; Accepted September 18, 2013

DOI: $10.3892 / \mathrm{mmr} .2013 .1716$

\begin{abstract}
Cip1-interacting zinc finger protein 1 (CIZ1) is a nuclear protein that was observed to bind to $\mathrm{p} 21^{\mathrm{Cip} 1 / \mathrm{Waf} 1}$. p $21^{\text {Cip1/Waf } 1}$ regulates the cell cycle and is associated with colorectal cancer (CRC) progression. However, the effect of CIZ1 on CRC cells remains unclear. In the present study, CIZ1 was observed to be highly expressed in RKO human CRC cells. Silencing of CIZ1 using small interfering RNA (siRNA) suppressed RKO cell proliferation. Flow cytometric analysis demonstrated that knockdown of CIZ1 decreased the percentage of cells in the $S$ phase and increased the ratio of cells in the G0/G1 phase in parallel with upregulated cell apoptosis. Moreover, the number and size of RKO cell colonies was repressed by knockdown of the $C I Z 1$ gene. These results suggested that CIZ1 may be involved in colon cancer progression by regulating cell proliferation, cell cycle, apoptosis and colony formation. Furthermore, CIZ1-siRNA may provide a novel tool for CRC investigation and therapy.
\end{abstract}

\section{Introduction}

Colorectal carcinoma (CRC) is one of the most common types of malignant gastrointestinal tumors. It is the third most

Correspondence to: Professor Xigui Yang, Department of Internal Medicine Oncology, Shandong Cancer Hospital, Shandong Academy of Medical Sciences, 440 Jiyan Road, Jinan, Shandong 250117, P.R. China

E-mail: zihuadiding80@163.com

Professor Xun Qu, Institute of Basic Medical Sciences, Qilu Hospital, Shandong University, 107 Wenhuaxi Road, Jinan, Shandong 250012, P.R. China

E-mail:quxun@sdu.edu.cn

Key words: Cip1 interacting zinc finger protein, colorectal cancer cells, proliferation, apoptosis common type of cancer in the United States (1) and affects $\sim 1$ million people each year, with a 5-year survival rate of $62 \%$. Although surgery, radiotherapy and chemotherapy have been widely used in the treatment of CRC, they are limited in preventing the progression of the disease and reducing mortality rates. Advances in human genome studies have revealed that gene alterations are key in the oncogenesis and progression of CRC $(2,3)$.

Cip1 interacting zinc finger protein 1 (CIZ1) is a nuclear protein and is encoded by 17 exons that map to chromosome $9 q 34$ (4). It was initially isolated in 1999 (5) and is reported to be important in mammalian DNA replication and transcription (6). CIZ1 was previously shown to be expressed in several cancer cell lines, including HeLa cells and ZR-75 human breast ductal carcinoma cells, and was suggested to be involved in the pathogenesis of lung cancer, lymphoma, pancreatic cancer and a number of estrogen-related tumors $(7,8)$. In addition, CIZ1 was observed to bind to the N-terminal region of $\mathrm{p} 21^{\text {Cip1/Waf1 }}$ through the first zinc finger motif and to subsequently result in translocation from the nucleus to the cytoplasm. p21 Cip1/Waf 1 is a downstream target of the P53 tumor suppressor gene (9) and has been suggested to be one of the primary causes of CRC progression $(9,10)$. However, the involvement of CIZ1 in human CRC has not previously been addressed.

In the current study, CIZ1 expression in several human CRC cell lines was examined and the effect of CIZ1 on cell proliferation, cell cycle and apoptosis of one of these lines, RKO, was investigated.

\section{Materials and methods}

Cell culture. Five CRC cell lines, RKO, DLD-1, HCT116, SW620 and LOVO, were obtained from American Type Cell Culture (ATCC; Manassas, VA, USA) and cultured in ATCC-recommended media in a humidified incubator at $5 \% \mathrm{CO}_{2}$ and $37^{\circ} \mathrm{C}$. For lentiviral transfection, RKO cells were resuspended in $0.25 \%$ trypsin and plated in six-well plates $(2,000$ cells/well). For colony formation, RKO cells 
were allowed to proliferate for 14 days. Following washing with phosphate-buffered saline (PBS), cells were treated with Giemsa for $20 \mathrm{~min}$ and washed three times with $\mathrm{ddH}_{2} \mathrm{O}$. Images were captured with a fluorescence microscope (Micropublisher 3.3RTV; Olympus Corporation, Shibuya, Japan) and the colonies were counted.

Transfection. Small interfering RNA (siRNA) sequences targeting human CIZ1 (GenBank accession no. GCG-291708) were designed by Genechem Co., Ltd. (Shanghai, China) using the following template: GCACTTAGTGCTGCAACAGAA. Following confirmation by sequencing, the sequences were cloned into a pGCSIL-GFP vector (GeneChem, Shanghai, China) to generate CIZ1-siRNA lentiviral vectors, which were then used to infect RKO cells. The CIZ1 lentiviral vector-transfected and non-transfected cells were included as a control. CIZ1 expression was measured by green fluorescent protein (GFP) expression using fluorescence microscopy (Micropublisher 3.3RTV)three days following infection; and the knockdown efficiency was measured by quantitative PCR (qPCR) and western blot analysis 5 days following infection.

$q P C R$. Total RNA was isolated by the TRIzol method (Invitrogen Life Technologies, Carlsbad, CA, USA). First-strand cDNA was generated by two-step qPCR using the following primers: Forward: 5'-GCCAAACAATCC TTGCGAC-3' and reverse: 5'-CAACCCACAGCGTCC ACT-3' for CIZI; and forward: 5'-TGACTTCAACAGCG ACACCCA-3' and reverse: 5'-CACCCTGTTGCTGTAGC CAAA-3' for GAPDH. qPCR comprised of an initial denaturation step at $95^{\circ} \mathrm{C}$ for $15 \mathrm{sec}, 45$ cycles at $95^{\circ} \mathrm{C}$ for $5 \mathrm{sec}$ and $60^{\circ} \mathrm{C}$ for $30 \mathrm{sec}$. Results are presented as $\mathrm{Ct}$ values, defined as the threshold PCR cycle number at which an amplified product was first detected. The average $\mathrm{Ct}$ was calculated for $C I Z 1$ and $G A P D H$ and ${ }^{\triangle} \mathrm{Ct}$ was determined as the mean of the triplicate $\mathrm{Ct}$ values for $C I Z 1$ minus the mean of the triplicate $\mathrm{Ct}$ values for $G A P D H$. The $2^{-(\Delta \Delta \mathrm{Ct})}$ method was used to analyze the relative changes in gene expression. All samples were examined at least three times.

Western blot analysis. Cells were harvested in RIPA lysis buffer that was supplemented with protease and phosphatase inhibitor cocktails (Shanghai Chemical Reagent Co., Ltd., Shanghai, China). Proteins were separated by sodium dodecyl sulfate-polyacrylamide gel electrophoresis, transferred onto polyvinylidene fluoride membranes and incubated with the following antibodies: Anti-CIZ1 (1:3,000; Sigma-Aldrich St. Louis, MO, USA) and anti-GAPDH (1:5,000; Santa-Cruz Biotechnology, Inc., Santa Cruz, CA, USA). Secondary antibodies conjugated to horseradish peroxidase and enhanced chemiluminescence western blotting reagents were used for detection.

Cellomics method. Cellomics Whole Cell Stains (Thermo Scientific, Waltham, MA, USA) provide excellent staining for high content screening (HCS) assays and fluorescence microscopy (Micropublisher 3.3RTV). Cell growth was measured via multiparametric HCS. Briefly, RKO cells at 10 days after infection with either CIZ1-siRNA lentivirus and NC lentivirus were seeded at 2,000 cells per well in 96-well plates, then incubated at $37^{\circ} \mathrm{C}$ with $5 \% \mathrm{CO}_{2}$ for five days. Plates were processed with the ArrayScan HCS software (Thermo Scientific) and kept at $+4^{\circ} \mathrm{C}$ for up to $24 \mathrm{~h}$ before each day's analysis. The system is a computerized, automated fluorescence-imaging microscope that automatically identifies stained cells and reports the intensity and distribution of fluorescence in each individual cell. Images were acquired for each fluorescence channel, using suitable filters and a 20x objective. In each well, at least 800 cells were analyzed. Images and data were stored in a Microsoft SQL database (Microsoft, Remond, WA, USA) for easy retrieval.

Flow cytometry. Cells were collected when the cell density had reached $80 \%$. For cell cycle detection, cells were washed twice with ice-cold PBS, fixed with $70 \%$ ice-cold ethanol and stained with propidium iodide. For cell apoptosis analysis, cells were stained with Annexin V-APC at room temperature in the dark for 10-15 min and detected by flow cytometry (FACSCalibur; Becton Dickinson, Franklin Lakes, NJ, USA). Cells were analyzed using flow cytometry. All experiments were repeated at least three times.

Statistical analysis. Values are expressed as the mean \pm SEM of these observations. Statistical analysis was performed using SPSS software (Release 13.0, SPSS Inc., Chicago, IL, USA). The difference between the two groups was analyzed by Student's t-test. $\mathrm{P}<0.05$ was considered to indicate a statistically significant difference.

\section{Results}

CIZ1 was highly expressed in RKO CRC cells and was successfully knocked down by CIZ1-siRNA. The expression of CIZ1 was clearly detected in all five CRC cell lines examined (RKO, DLD-1, HCT116, SW620 and LOVO cells; Fig. 1A). Among these, the expression level of CIZ1 in RKO cells was significantly higher compared with the other cell lines; thus, RKO cells were used for subsequent experiments. To investigate the effect of CIZ1 on RKO cells, a CIZ1-siRNA-GFP lentivirus was generated and RKO cells were transfected to knockdown $C I Z 1$ gene expression. To verify the knockdown efficiency of CIZ1, we detected its mRNA expression in RKO cells by qPCR. CIZ1 expression was observed to be significantly reduced compared with the negative control that was transfected with $C I Z 1$ alone $(\mathrm{P}<0.05$; Fig. 1B). This result was further confirmed by the detection of CIZ1 protein expression by western blot analysis (Fig. 1C), demonstrating that the CIZ1 gene was efficiently knocked down by CIZ1-siRNA. CIZ1 expression was also visualized by GFP expression under fluorescent microscopy three days following infection (Fig. 1D), $>80 \%$ of RKO cells expressed GFP. Under light microscopy (XSP-8CA; Shanghai Optical Instrument Factory, Shanghai, China), no significant difference was identified between the negative control and the non-transfected groups, indicating that the lentivirus itself and the transfection process had no effect on cell proliferation (Fig. 1D).

Knockdown of CIZ1 inhibits RKO cell proliferation, induces cell cycle arrest and increases apoptosis. The effect of CIZ1 expression on RKO cell proliferation was examined. Cells 
A

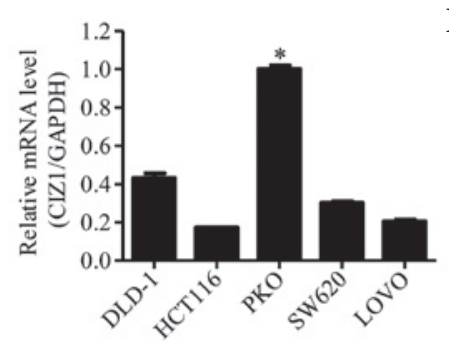

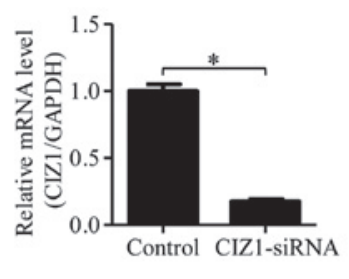

C

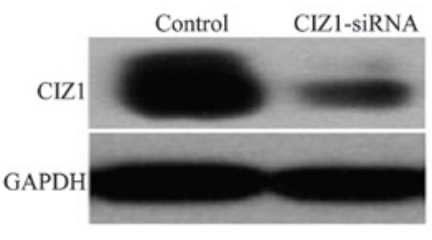

D

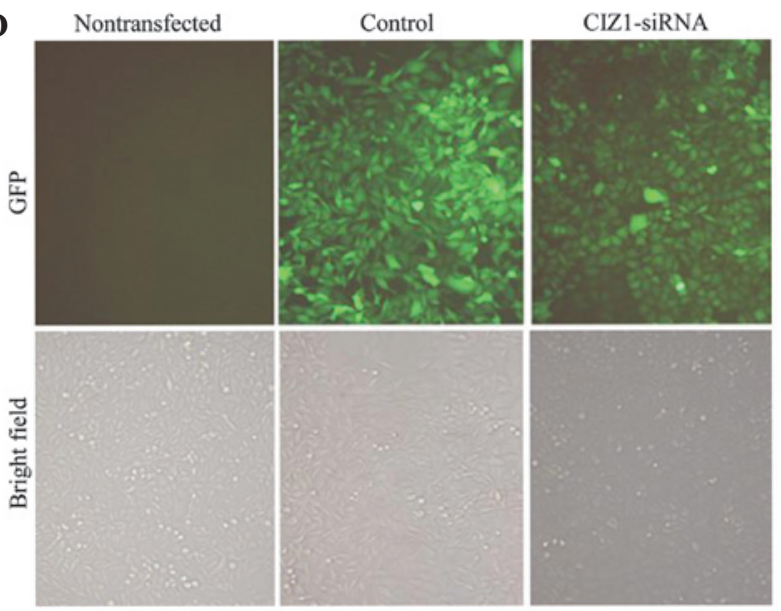

Figure 1. CIZ1 expression in colorectal cancer (CRC) cell lines and knockdown of the CIZ1 gene in RKO cells. The expression of CIZ1 mRNA in five CRC cell lines was measured by quantitative PCR (qPCR). (A) The y-axis shows CIZ1 levels relative to that of an internal control, the glyceraldehyde 3-phosphate dehydrogenase $(G A P D H)$ gene $\left({ }^{*} \mathrm{P}<0.05\right.$, vs. the other groups). RKO cells were infected with a lentivirus containing a CIZ1-small interfering RNA (siRNA) insertion. CIZ1 expression was measured by (B) qPCR and (C) western blot analysis ( $\mathrm{P}<0.05$, vs. control) and visualized as green fluorescent protein (GFP) cells under a fluorescence microscope (D). RKO cells transfected with lentivirus alone served as a control. CIZ1, Cip1 interacting zinc finger protein 1.

A
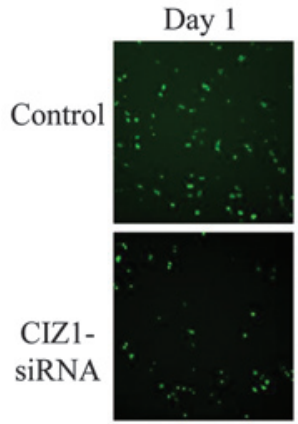

Day 2
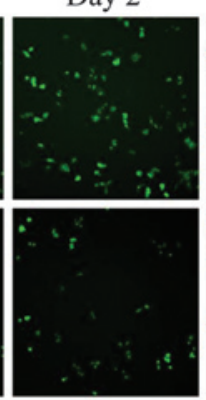

Day 3
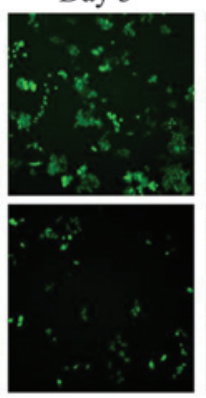

Day 4
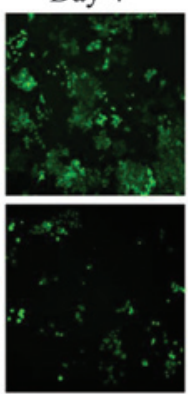

Day 5

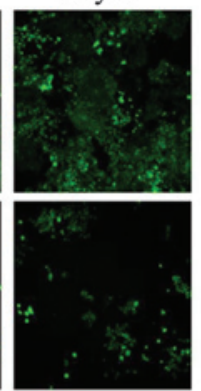

B

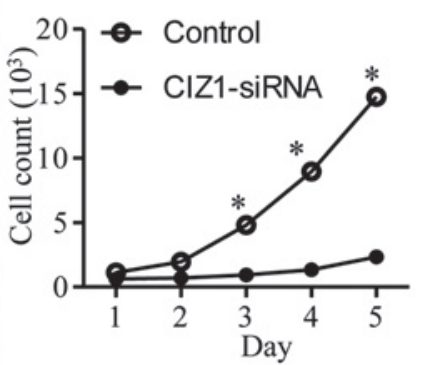

Figure 2. Knockdown of the CIZ1 gene kinetically inhibits RKO cell proliferation. (A) CIZ1-siRNA-targeted RKO cells and the negative control were observed by the Cellomics method for five days. (B) Cell proliferation was significantly inhibited in CIZ1-siRNA-transfected cells compared with the negative control $($ P $<0.05$, vs. control). CIZ1, Cip1 interacting zinc finger protein 1.

transfected with CIZ1-siRNA lentivirus and lentivirus alone were resuspended in $0.25 \%$ trypsin and replicates were plated in five wells of six-well plates to equalize the results. Cells were counted once daily for five days using the Cellomics method (Fig. 2A). Cells from the two groups divided slowly for the first two days. However, on day three, a significantly increased number of cells were counted in the control group compared with the CIZ1-siRNA group and the effect was more pronounced on days four and five (Fig. 2B; $\mathrm{P}<0.05$ ), indicating that knockdown of CIZ1 may significantly inhibit the proliferation of RKO cells. In addition, the effect of CIZ1 on cell cycle distribution was investigated using flow cytometry. The percentage of G0/G1 stage cells was increased in the CIZ1-siRNA group $(46.11 \pm 0.64)$ compared with the control group $(42.66 \pm 0.75 ; \mathrm{P}=0.003)$, while the percentage of cells in the $\mathrm{S}$ phase was decreased $(33.91 \pm 0.58)$ compared with the control group $(36.83 \pm 1.05)(\mathrm{P}=0.02$; Fig. $3 \mathrm{~A}$ and $\mathrm{B})$. These data suggested that CIZ1 blocked the progression of the cell cycle. Furthermore, the effect of CIZ1 on RKO cell apoptosis was examined. Cell were stained with Annexin V and detected by flow cytometry. Knockdown of the CIZ1 gene markedly increased the percentage of apoptotic cells (Fig. 3C and D).

Knockdown of CIZ1 repressed RKO cell colony formation. To investigate the effect of CIZ1 on colony formation, RKO cells were allowed to proliferate for 14 days to form colonies in six-well plates. Colonies were stained with Giemsa, counted 
A

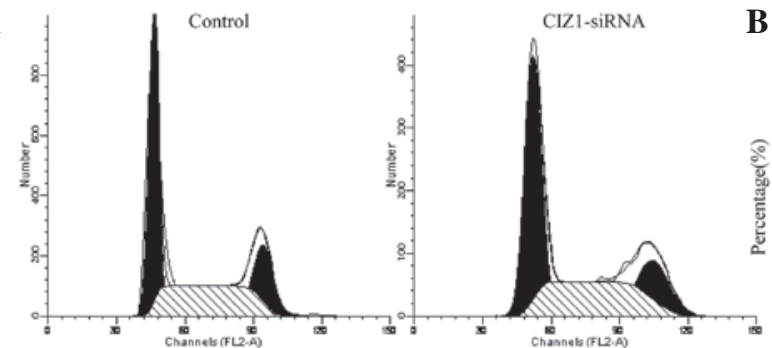

B

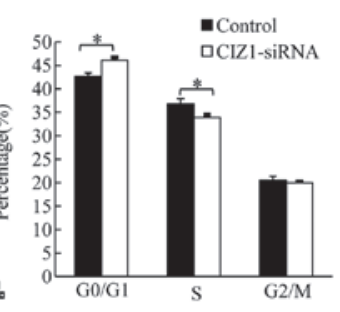

C

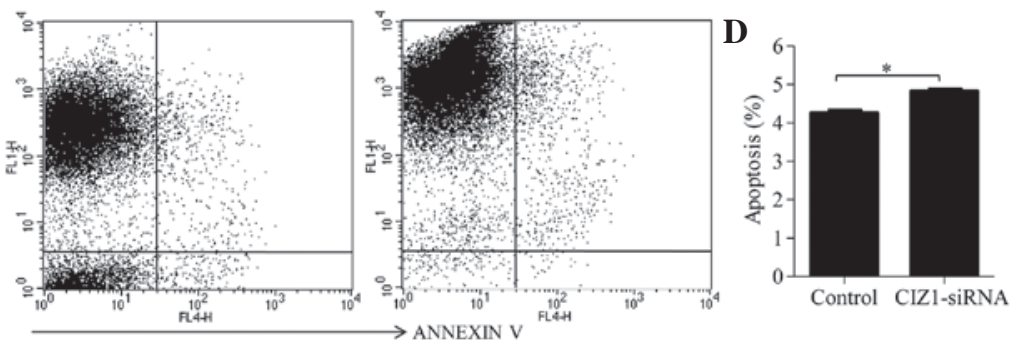

Figure 3. Knockdown of the CIZ1 gene induces cell cycle arrest and increases RKO apoptosis. (A) Cell cycle distribution was analyzed by flow cytometry. (B) The bar graph indicates the percentages of cells at G0/G1, S and G2/M stage (" $\mathrm{P}<0.05$, vs. control). (C) Apoptotic cells were stained with Annexin V and detected by flow cytometry. (D) The percentages of apoptotic cells in each culture system. Data represent at least three experiments ("P<0.05, vs. control). CIZ1, Cip1 interacting zinc finger protein 1.
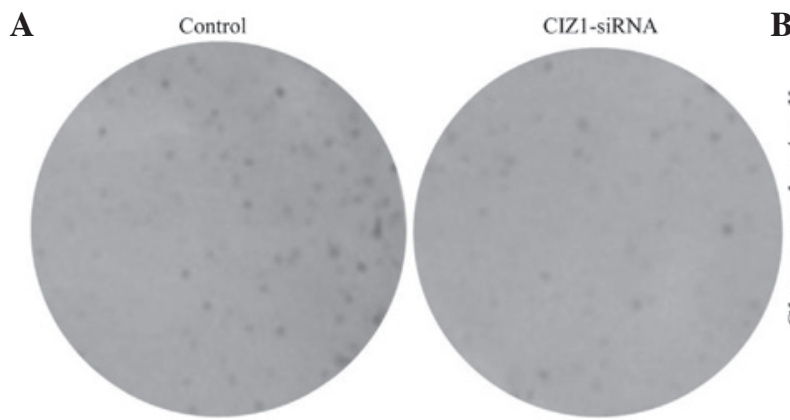

B

C

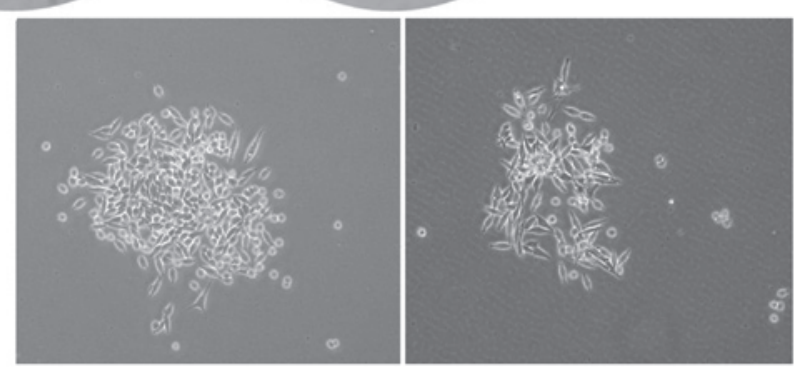

Figure 4. Knockdown of the CIZ1 gene inhibits colony formation of RKO cells. Lentivirus-infected RKO cells were allowed to grow for 14 days in six-well plates. (A) Photomicrographs of stained colonies. (B) The number of colonies in each group ("P $<0.01$, vs. control). (C) Representative colonies. CIZ1, Cip1 interacting zinc finger protein 1.

and analyzed. CIZ1-siRNA lentiviral infection markedly reduced the colonies of RKO cells by $69.0 \%$, compared with the control lentivirus-infected groups ( $\mathrm{P}<0.01$; Fig. $4 \mathrm{~A}$ and $\mathrm{B})$. Moreover, the colonies of the CIZ1-siRNA-infected group were also smaller and more dispersed compared with the control group (Fig. 4C).

\section{Discussion}

In this study, the expression levels of CIZI mRNA in human CRC cell lines and their function in a human CRC cell line, RKO, were investigated. The results demonstrated that knockdown of CIZ1 using CIZ1-siRNA inhibited RKO cell proliferation, induced cell cycle arrest, increased apoptosis and eventually repressed RKO cell colony formation.

CIZ1, as a DNA replication regulator, was initially characterized to interact with $\mathrm{p} 21^{\text {Cipl/Waf1 }}(5)$. p $21^{\text {Cip//Wafl }}$ is induced by the P53 tumor suppressor protein and is a cell cycle regulator (12). Previous studies showed that $\mathrm{p} 21^{\mathrm{Cip} / / \text { Wafl }}$ inhibits cell cycle progression by binding to G1 cyclin/cyclin-dependent kinase (CDK) complexes and proliferating cell nuclear antigen through its $\mathrm{N}$ - and $\mathrm{C}$-terminal domains, respectively $(5,13)$. Therefore, $\mathrm{p} 21^{\mathrm{Cip} 1 / \mathrm{Waf} 1}$ has been suggested to be critical in inhibiting progression of cancer cells, including fibroblasts, $\mathrm{HaCaT}$ and breast cancer cells $(14,15)$. CIZ1 was observed to bind to $\mathrm{p} 21^{\mathrm{Cip} 1 / \text { Waf1 }}$, competitively inhibiting the interaction of 
p2 $1^{\text {Cip1/Waf1 }}$ with CDK and subsequently regulating cytoplasmic distribution (5). Therefore, it is reasonable to assume that CIZ1 regulates tumor cell progression by affecting the cell cycle. The current study demonstrated that knockdown of CIZ1 gene inhibits RKO cell proliferation and promotes cell cycle arrest.

Further studies are required to determine the mechanism by which CIZ1-siRNA inhibits RKO cell proliferation as CIZ1 is also a DNA replication factor as well as an inhibitor of $\mathrm{p} 21^{\text {Cip1/Waf1 }}$. Normally, CIZ1 is attached to the nuclear matrix and resides within foci that partially colocalize at sites of DNA replication (16). CIZ1 promotes the initiation of mammalian DNA replication by coordinating the sequential functions of cyclin E- and A-dependent protein kinases $(17,18)$. It is also indirectly involved in DNA replication by modulating the expression of genes, including cyclin D, that affect cell proliferation (6). Therefore, it is also possible that CIZ1 regulates RKO cell proliferation, cell cycle and apoptosis by affecting DNA replication, which is a $\mathrm{p} 21^{\mathrm{Cip} 1 / \text { Waf } 1}$ independent pathway.

The present study showed that knockdown of $C I Z 1$ expression negatively controlled the progression of RKO CRC cells in vitro. These results indicate that CIZ1-siRNA may be a promising therapeutic strategy for CRC.

\section{Acknowledgements}

This study was funded by a grant from the Shandong Provincial Natural Science Foundation (grant no. ZR2010HQ055).

\section{References}

1. Kaur M, Mandair R, Agarwal R and Agarwal C: Grape seed extract induces cell cycle arrest and apoptosis in human colon carcinoma cells. Nutr Cancer 60 (Suppl 1): 2-11, 2008.

2. Tomlinson IP, Webb E, Carvajal-Carmona L et al: A genomewide association study identifies colorectal cancer susceptibility loci on chromosomes 10p14 and 8q23.3. Nat Genet 40: 623-630, 2008.

3. Tenesa A, Farrington SM, Prendergast JG et al: Genome-wide association scan identifies a colorectal cancer susceptibility locus on 11q23 and replicates risk loci at $8 \mathrm{q} 24$ and 18q21. Nat Genet 40, 631-637, 2008.
4. Rahman FA, Aziz N and Coverley D: Differential detection of alternatively spliced variants of Cizl in normal and cancer cells using a custom exon-junction microarray. BMC Cancer 10: 482, 2010.

5. Mitsui K, Matsumoto A, Ohtsuka S, Ohtsubo M and Yoshimura A: Cloning and characterization of a novel p21(Cip1/Waf1)-interacting zinc finger protein, ciz1. Biochem Biophys Res Commun 264: 457-464, 1999.

6. den Hollander P, Rayala SK, Coverley D and Kumar R: Ciz1, a novel DNA-binding coactivator of the estrogen receptor alpha, confers hypersensitivity to estrogen action. Cancer Res 66: 11021-11029, 2006.

7. Warder DE and Keherly MJ: Ciz1, Cip1 interacting zinc finger protein 1 binds the consensus DNA sequence ARYSR(0-2) YYAC. J Biomed Sci 10: 406-417, 2003.

8. Rahman F, Ainscough JF, Copeland N and Coverley D: Cancer-associated missplicing of exon 4 influences the subnuclear distribution of the DNA replication factor CIZ1. Hum Mutat 28: 993-1004, 2007.

9. Stein GH, Drullinger LF, Soulard A and Dulić V: Differential roles for cyclin-dependent kinase inhibitors p21 and p16 in the mechanisms of senescence and differentiation in human fibroblasts. Mol Cell Biol 19: 2109-2117, 1999.

10. Kinzler KW and Vogelstein B: Lessons from hereditary colorectal cancer. Cell 87: 159-170, 1996.

11. Samowitz WS, Curtin K, Ma KN, Edwards S, Schaffer D, Leppert MF and Slattery ML: Prognostic significance of p53 mutations in colon cancer at the population level. Int J Cancer 99: 597-602, 2002

12. Poon RY and Hunter T: Expression of a novel form of p21Cip1/Waf1 in UV-irradiated and transformed cells. Oncogene 16: 1333-1343, 1998.

13. Lim DY, Tyner AL, Park JB, Lee JY, Choi YH and Park JH: Inhibition of colon cancer cell proliferation by the dietary compound conjugated linoleic acid is mediated by the CDK inhibitor p21CIP1/WAF1. J Cell Physiol 205: 107-113, 2005.

14. Wei M, Liu B, Gu Q, Su L, Yu Y and Zhu Z: Stat6 cooperates with $\mathrm{Sp} 1$ in controlling breast cancer cell proliferation by modulating the expression of p21(Cip1/WAF1) and p27 (Kip1). Cell Oncol (Dordr) 36: 79-93, 2013.

15. Zamkova M, Khromova N, Kopnin BP and Kopnin P: Ras-induced ROS upregulation affecting cell proliferation is connected with cell type-specific alterations of HSF1/SESN3/p21Cip1/WAF1 pathways. Cell Cycle 12: 826-836, 2013.

16. Ainscough JF, Rahman FA, Sercombe H, Sedo A, Gerlach B and Coverley D: C-terminal domains deliver the DNA replication factor Ciz1 to the nuclear matrix. J Cell Sci 120: 115-124, 2007.

17. Coverley D, Marr J and Ainscough J: Ciz1 promotes mammalian DNA replication. J Cell Sci 118: 101-112, 2005.

18. Copeland NA, Sercombe HE, Ainscough JF and Coverley D: Ciz1 cooperates with cyclin-A-CDK2 to activate mammalian DNA replication in vitro. J Cell Sci 123: 1108-1115, 2010. 